April 2008

\title{
Mirror dark matter and the new DAMA/LIBRA results: A simple explanation for a beautiful experiment
}

\author{
R. Foot ${ }^{1}$ \\ School of Physics, \\ University of Melbourne, \\ Victoria 3010 Australia
}

Recently, the DAMA/LIBRA experiment has convincingly confirmed the DAMA/NaI annual modulation signal, experimentally demonstrating the existence of non-baryonic dark matter in the halo of our galaxy. Meanwhile, in another part of town, other experiments such as CDMS and XENON10 have not detected any evidence for dark matter. One promising dark matter candidate which can reconcile the positive DAMA annual modulation signal with the null results from the other experiments, is mirror dark matter. We re-analyse the mirror matter interpretation of the DAMA annual modulation signal utilizing a) the new data from DAMA/LIBRA, including the measured energy dependence of the annual modulation signal b) an updated quenching factor which takes into account the channeling effect in $\mathrm{NaI}$ crystals and c) the latest constraints from CDMS/Ge, CDMS/Si and XENON10 experiments. We show that the simplest possibility of a $H e^{\prime}$ (and/or $H^{\prime}$ ) dominated halo with a small $O^{\prime}$ component is sufficient to fully explain all of the dark matter experiments. We also point out that a certain class of hidden sector dark matter models, although theoretically less appealing and less constrained, can mimic the success of the mirror dark matter model and hence are also viable.

\footnotetext{
${ }^{1}$ E-mail address: rfoot@unimelb.edu.au
} 


\section{Introduction}

Recently the DAMA/LIBRA experiment has confirmed[1] the impressive DAMA/NaI annual modulation signal at around $8 \sigma$ C.L.[2]. Their signal is observed in the $2-6$ keVee energy region, with periodicity and phase:

$$
T / \text { year }=0.998 \pm 0.003, t_{0} / \text { day }=144 \pm 8,
$$

which is beautifully consistent with the dark matter expectation of $T /$ year $=1.0$, $t_{0} /$ day $=152.5$. There are no known systematic effects which could produce the modulation of the signal seen. Indeed, if there were some hypothetical systematic effect producing the modulation, it would be surprising if it had the same periodicity AND phase as the dark matter signal. Therefore, it is reasonable to believe that these experiments have detected galactic dark matter particles.

Meanwhile other experiments have failed to detect positive evidence for dark matter. Among the most sensitive of these, are the recent CDMS/Ge[3], CDMS/Si[4] and XENON10[5] experiments. Combined analysis of these null results with the positive results of DAMA appear to exclude standard WIMP explanations (such as the standard neutralino models) for the DAMA signal. Several alternative explanations of the DAMA signal have been proposed, such as the elastic scattering of light WIMPs (with mass $7 \mathrm{GeV}$ )[6], and very light candidates (e.g. with mass in the keV range) scattering off electrons $[7,8]$. While such phenomological models might be possible, it is a theoretical challenge to construct simple renormalizable extensions to the standard model which can accommodate these light particles.

On the other hand, previous work $[9,10,11,12]$ has shown that mirror dark matter can explain the DAMA/NaI annual modulation signal, consistently with the null results of the other experiments. Mirror dark matter arises in simple renormalizable extensions of the standard model featuring a sector of particles and forces exactly isomorphic (or "mirror") to the known particles and forces. The simplest such model being the exact parity symmetric model[14] (for a review, see e.g. ref.[13]). These theories feature a spectrum of dark matter particles, $A^{\prime}$, with known masses.

The success of the mirror dark matter theory in explaining the DAMA/NaI experiment consistently with the null results of the other dark matter detection experiments has to do with several of its key distinctive features:

- Galactic halo mirror particles have a Maxwellian velocity distribution: $f(u)=$ $\exp \left[-u^{2} / v_{0}^{2}\right]$, however the velocity dispersion of heavy mirror particles $\left(M_{A^{\prime}}>\right.$ $\left.M_{H e}\right)$ is much less than the galactic rotational velocity: $v_{0}\left(A^{\prime}\right)^{2} \ll v_{r o t}^{2}$. In standard WIMP models, $v_{0}^{2}=v_{\text {rot }}^{2}$.

- The mirror dark matter mass spectrum consists of relatively light particles: $M_{A^{\prime}} \leq$ $M_{F e}$. Standard WIMP models typically feature $M_{W I M P} \gtrsim 50 \mathrm{GeV}$.

- Mirror dark matter interacts with ordinary matter via renormalizable photonmirror photon kinetic mixing, leading to a recoil energy $\left(E_{R}\right)$ dependent crosssection: $\frac{d \sigma}{d E_{R}} \propto \frac{1}{E_{R}^{2}}$. Standard WIMP models have an approximately $E_{R}$ independent cross-section arising from a four-Fermion interaction. 
These three features greatly increase the sensitivity of mirror dark matter experiments such as DAMA/NaI. The main reason is that the DAMA/NaI experiment is a lower threshold experiment than the sensitive CDMS and Xenon experiments, when the channeling effect is taken into account[15].

The purpose of this paper is to re-analyse the mirror matter interpretation of the DAMA annual modulation signal in the light of the new data from DAMA/LIBRA as well as the latest constraints from CDMS and XENON experiments. We also use an updated quenching factor, taking into account the channeling effect in $\mathrm{NaI}$ crystals, which was discussed recently by the DAMA collaboration[15].

The outline of this paper is as follows: in section II we give a brief review of mirror dark matter, which may be skipped by those already familiar with the subject. In section III, we give the necessary details relevant to direct detection experiments such as cross-section, halo distribution function etc. In section IV we give the detailed fit to the DAMA/LIBRA + DAMA/NaI combined results under the simple and plausible assumption that the heavy component of the galactic halo (i.e. with $M>M_{\mathrm{He}}$ ) is dominated by just one mirror element. That is, one element in addition to the $H^{\prime} / H e^{\prime}$ predominant component. In section $\mathrm{V}$ we examine the constraints imposed by the negative results of the CDMS/Ge, CDMS/Si and XENON10 experiments. In section VI we point out that a class of hidden sector models can mimic the success of the mirror dark matter theory in explaining the experiments. Finally, in section VII we draw our conclusions.

\section{Mirror Dark Matter}

The exact parity symmetric model[14] is the minimal extension of the standard model which allows for an exact unbroken parity symmetry $[x \rightarrow-x, t \rightarrow t]$. According to this theory, each type of ordinary particle (electron, quark, photon etc) has a corresponding mirror partner (mirror electron, mirror quark, mirror photon etc), of the same mass. The two sets of particles form parallel sectors each with gauge symmetry $G$ (where $G=S U(3) \otimes S U(2) \otimes U(1)$ in the simplest case) so that the full gauge group is $G \otimes$ $G$. The unbroken mirror symmetry maps $x \rightarrow-x$ as well as ordinary particles into mirror particles. Exact unbroken time reversal symmetry also exists, with standard CPT identified as the product of exact $\mathrm{T}$ and exact $\mathrm{P}[14]$.

It has been argued that the stable mirror particles, mirror nucleons and mirror electrons are an interesting candidate for the inferred dark matter of the Universe (for a review, see Ref.[13]). Of course, to be a successful dark matter candidate, mirror matter needs to behave, macroscopically, differently to ordinary matter. In particular, four key distinctions need to be explained:

- The cosmological abundance of mirror matter should be different to ordinary matter, $\Omega_{\text {dark }} \neq \Omega_{\text {matter }}$.

- Mirror particles should give negligible contribution to the energy density at the epoch of big bang nucleosynthesis. 
- Structure formation in the mirror sector must begin before ordinary matter radiation decoupling.

- In spiral galaxies, the time scale for the collapse of mirror matter onto the disk must be much longer than that of ordinary matter.

Clearly, mirror matter behaves differently to ordinary matter, at least macroscopically. It is hypothesised that this macroscopic asymmetry originates from effectively different initial conditions in the two sectors. The exact microscopic (Lagrangian) symmetry between ordinary and mirror matter need never be broken. In particular, if ordinary and mirror particles have different temperatures in the early Universe, $T^{\prime} \ll T{ }^{2}$ then the mirror particles give negligible contribution to the energy density at the time of nucleosynthesis leading to standard big bang nucleosynthesis. Another consequence of $T^{\prime} \ll T$ is that mirror photon decoupling occurs earlier than ordinary photon decoupling - implying that mirror structure formation can begin before ordinary photon decoupling. In this way, mirror matter-type dark matter can successfully explain the large scale structure formation (for detailed studies, see ref.[17, 18, 19]). Also, $\Omega_{\text {dark }} \neq \Omega_{\text {matter }}$ could also be due to different effective initial conditions in the early Universe (see e.g. ref.[20],[21] for some specific scenarios).

If mirror matter is the inferred non-baryonic dark matter in the Universe, then the halo of our galaxy should be a gas of ionized mirror atoms and mirror electrons with a possible mirror star component ${ }^{3}$. Although dissipative, roughly spherical galactic mirror matter halo's can exist without collapsing provided that a heating mechanism exists - with ordinary supernova explosions being plausible candidates[24]. Obviously, the heating of the ordinary and mirror matter in spiral galaxies needs to be asymmetric, but again, due to different initial conditions in the early Universe, asymmetric heating is plausible. For example, the early Universe temperature asymmetry, $T^{\prime} \ll T$ (expected from successful big bang nucleosynthesis and Large scale structure formation, as discussed above) implies that the primordial mirror helium/mirror hydrogen ratio will be much larger than the corresponding ordinary helium/ordinary hydrogen ratio[17]. Consequently the formation and evolution of stars in the ordinary and mirror sectors are completely different (see e.g. ref.[25] for some preliminary studies). The details of the evolution on (sub) galactic scales, is of course, very complex, and is yet to be fully understood.

Ordinary and mirror particles interact with each other by gravity and via renormalizable photon-mirror photon kinetic mixing: ${ }^{4}$

$$
\mathcal{L}=\frac{\epsilon}{2} F^{\mu \nu} F_{\mu \nu}^{\prime}
$$

\footnotetext{
${ }^{2}$ It has been speculated that $T^{\prime} \ll T$ might arise in certain inflation scenarios, see e.g. ref.[16].

${ }^{3}$ The mirror star component can be probed by microlensing observations towards nearby galaxies[22]. The current situation is somewhat unclear, but a mass fraction of MACHO's around $f \sim 0.1$ is roughly compatible with the observations. For a review, see ref.[23]

${ }^{4}$ Technically, photon-mirror photon kinetic mixing arises from kinetic mixing of the $U(1)$ and $U(1)^{\prime}$ gauge fields, since only for the abelian $U(1)$ gauge symmetry is such mixing gauge invariant[26]. The only other gauge invariant and renormalizable interactions mixing ordinary and mirror particles are the quartic Higgs - mirror Higgs interaction: $\lambda \phi^{\dagger} \phi \phi^{\prime \dagger} \phi^{\prime}$ and neutrino - mirror neutrino mass mixing[14, 27].
} 
where $F^{\mu \nu}\left(F_{\mu \nu}^{\prime}\right)$ is the field strength tensor for electromagnetism (mirror electromagnetism). One effect of photon-mirror photon kinetic mixing is to cause mirror charged particles (such as the mirror proton and mirror electron) to couple to ordinary photons with effective electric charge $\epsilon e .[14,28,29]$ The various experimental implications of photon-mirror photon kinetic mixing have been reviewed in Ref.[30]. Of most relevance for this paper, is that this interaction enables mirror particles to elastically scatter off ordinary particles - essentially Rutherford scattering.

A detector on Earth can therefore be used to detect halo mirror nuclei via elastic scattering. Several previous papers $[9,10,11,12]$ have explored this possibility, especially in view of the impressive dark matter signal from the DAMA/NaI experiment. The purpose of this paper is to re-analyse the mirror matter interpretation of the DAMA/NaI experiment using the new data from DAMA/LIBRA and also the latest constraints from the null results of the CDMS and XENON10 experiments. In the following section we give the technical details - the cross section and halo distribution function - necessary to facilitate this comparison of theory and experiment.

\section{Mirror dark matter implications for direct detec- tion experiments}

\subsection{Cross-section and form factors}

Let us first briefly review the required technology (see ref. $[9,10,11,12]$ for more details). For definiteness, consider a halo mirror nuclei, $A^{\prime}$, of atomic number $Z^{\prime}$ scattering off an ordinary nucleus, $A$ (in an ordinary matter detector) of atomic number $Z$. The cross-section is then just of the standard Rutherford form corresponding to a particle of electric charge $Z e$ scattering off a particle of electric charge $\epsilon Z^{\prime} e$. This cross-section can be expressed in terms of the recoil energy of the ordinary atom, $E_{R}$, and the velocity of $A^{\prime}$ in the Earth's rest frame, $v$ :

$$
\frac{d \sigma}{d E_{R}}=\frac{\lambda}{E_{R}^{2} v^{2}}
$$

where

$$
\lambda \equiv \frac{2 \pi \epsilon^{2} \alpha^{2} Z^{2} Z^{\prime 2}}{M_{A}} F_{A}^{2}\left(q r_{A}\right) F_{A^{\prime}}^{2}\left(q r_{A^{\prime}}\right)
$$

and $F_{X}\left(q r_{X}\right)\left(X=A, A^{\prime}\right)$ are the form factors which take into account the finite size of the nuclei and mirror nuclei. $\left[q=\left(2 M_{A} E_{R}\right)^{1 / 2}\right.$ is the momentum transfer and $r_{X}$ is the effective nuclear radius $]^{5}$. A simple analytic expression for the form factor, which we adopt in our numerical work, is the one given by Helm[31, 32]:

$$
F_{X}\left(q r_{X}\right)=3 \frac{j_{1}\left(q r_{X}\right)}{q r_{X}} e^{-(q s)^{2} / 2}
$$

\footnotetext{
${ }^{5}$ We use natural units, $\hbar=c=1$ throughout.
} 
with $r_{X}=1.14 X^{1 / 3} \mathrm{fm}, s=0.9 \mathrm{fm}$. In this equation, $j_{1}$ is the spherical Bessel function of index 1 .

\subsection{Interaction rate and halo distribution function}

In an experiment such as DAMA/NaI, the measured quantity is the recoil energy, $E_{R}$, of a target atom. The interaction rate is

$$
\begin{aligned}
\frac{d R}{d E_{R}} & =\sum_{A^{\prime}} N_{T^{n}} n_{A^{\prime}} \int \frac{d \sigma}{d E_{R}} \frac{f_{A^{\prime}}\left(\mathbf{v}, \mathbf{v}_{E}\right)}{k}|v| d^{3} v \\
& =\sum_{A^{\prime}} N_{T^{n}} n_{A^{\prime}} \frac{\lambda}{E_{R}^{2}} \int_{|v|>v_{\min }\left(E_{R}\right)}^{\infty} \frac{f_{A^{\prime}}\left(\mathbf{v}, \mathbf{v}_{E}\right)}{k|v|} d^{3} v
\end{aligned}
$$

where $N_{T}$ is the number of target atoms per $\mathrm{kg}$ of detector ${ }^{6}$. Also, $n_{A^{\prime}}$ is the halo number density (at the Earth's location) of the mirror element, $A^{\prime}$ and $f_{A^{\prime}}\left(\mathbf{v}, \mathbf{v}_{E}\right) / k$ is its velocity distribution $\left(k \equiv\left(\pi v_{0}^{2}\right)^{3 / 2}\right.$ is the normalization factor). Here, $\mathbf{v}$ is the velocity of the halo particles relative to the Earth, and $\mathbf{v}_{E}$ is the Earth's velocity relative to the galactic halo. [Note that the bold font is used to indicate that the quantities are vectors]. The lower velocity limit, $v_{\min }\left(E_{R}\right)$, is given by the kinematic relation:

$$
v_{\text {min }}=\sqrt{\frac{\left(M_{A}+M_{A^{\prime}}\right)^{2} E_{R}}{2 M_{A} M_{A^{\prime}}^{2}}} .
$$

Considering a particular mirror chemical element, $A^{\prime}$ (e.g. $A^{\prime}=H^{\prime}, H e^{\prime}, O^{\prime}$ etc), the velocity distribution for these halo mirror particles is then:

$$
f_{A^{\prime}}\left(v, v_{E}\right)=\exp \left[-\frac{1}{2} M_{A^{\prime}}\left(\mathbf{v}+\mathbf{v}_{E}\right)^{2} / T\right]=\exp \left[-\left(\mathbf{v}+\mathbf{v}_{E}\right)^{2} / v_{0}^{2}\right]
$$

where $v_{0}^{2} \equiv 2 T / M_{A^{\prime}}$.

The assumption of approximate hydrostatic equilibrium for the halo particles implies a relation between $T$ and the local rotational velocity, $v_{\text {rot }}:[10]$

$$
T=\frac{\mu M_{p} v_{r o t}^{2}}{2}
$$

where $\mu M_{p}$ is the mean mass of the particles comprising the mirror (gas) component of the halo $\left(M_{p}\right.$ is the proton mass). Note that the Maxwellian distribution should be an excellent approximation in the case of mirror dark matter, since the self interactions of the particles ensure that the halo is locally thermalized.

The velocity integral in Eq.(6),

$$
I\left(E_{R}\right) \equiv \int_{|v|>v_{\min }\left(E_{R}\right)}^{\infty} \frac{f_{A^{\prime}}\left(\mathbf{v}, \mathbf{v}_{E}\right)}{k|v|} d^{3} v
$$

\footnotetext{
${ }^{6}$ For detectors with more than one target element we must work out the event rate for each element separately and add them up to get the total event rate.
} 
is standard (similar integrals occur in the usual WIMP interpretation ${ }^{7}$ ) and can easily be evaluated in terms of error functions assuming a Maxwellian dark matter distribution[32], $f_{A^{\prime}}\left(\mathbf{v}, \mathbf{v}_{E}\right) / k=\left(\pi v_{0}^{2}\right)^{-3 / 2} \exp \left[-\left(\mathbf{v}+\mathbf{v}_{E}\right)^{2} / v_{0}^{2}\right]$

$$
I\left(E_{R}\right)=\frac{1}{2 v_{0} y}[\operatorname{erf}(x+y)-\operatorname{erf}(x-y)]
$$

where

$$
x \equiv \frac{v_{\min }\left(E_{R}\right)}{v_{0}}, y \equiv \frac{v_{E}}{v_{0}} .
$$

The Earth's velocity relative to the galactic halo, $v_{E}$, has an estimated mean value of $\left\langle v_{E}\right\rangle \simeq v_{\text {rot }}+12 \mathrm{~km} / \mathrm{s}$, with $v_{\text {rot }}$, the local rotational velocity, in the $90 \%$ C.L. range[33],

$$
170 \mathrm{~km} / \mathrm{s} \lesssim v_{\text {rot }} \lesssim 270 \mathrm{~km} / \mathrm{s}
$$

While some estimates put narrower limits on the local rotational velocity, it is useful to allow for a broad range for $v_{r o t}$ since it can also approximate the effect of bulk halo rotation.

As can be seen from Eq. $(8,9)$, in the case of mirror matter-type dark matter, the $v_{0}$ value for a particular halo component element, $A^{\prime}$, depends on the chemical composition of the halo. In general,

$$
\frac{v_{0}^{2}\left(A^{\prime}\right)}{v_{\text {rot }}^{2}}=\frac{\mu M_{p}}{M_{A^{\prime}}}
$$

The most abundant mirror elements are expected to be $H^{\prime}, H e^{\prime}$, generated in the early Universe from mirror big bang nucleosynthesis (BBN). Heavier mirror elements are expected to be generated in mirror stars, or possibly, in the early universe if mirror BBN occurs early enough, so that the number densities are large enough, to allow three-body processes such as the triple alpha' process to be effective. It is useful, therefore, to consider two limiting cases: first that the halo is dominated by $H e^{\prime}$ and the second is that the halo is dominated by $H^{\prime}$. The mean mass of the particles in the halo are then (taking into account that the light halo mirror atoms should be fully ionized):

$$
\begin{aligned}
& \mu M_{p} \simeq M_{H^{\prime}} / 2 \simeq 0.5 \mathrm{GeV} \text { for } \mathrm{H}^{\prime} \text { dominated halo, } \\
& \mu M_{p} \simeq M_{H e^{\prime}} / 3 \simeq 1.3 \mathrm{GeV} \text { for } \mathrm{He}^{\prime} \text { dominated halo. }
\end{aligned}
$$

The $v_{0}$ values can then easily be obtained from Eq.(14):

$$
\begin{aligned}
& v_{0}\left(A^{\prime}\right)=v_{0}\left(H^{\prime}\right) \sqrt{\frac{M_{H^{\prime}}}{M_{A^{\prime}}}} \approx \frac{v_{r o t}}{\sqrt{2}} \sqrt{\frac{M_{H^{\prime}}}{M_{A^{\prime}}}} \mathrm{km} / \mathrm{s} \text { for } \mathrm{H}^{\prime} \text { dominated halo } \\
& v_{0}\left(A^{\prime}\right)=v_{0}\left(H e^{\prime}\right) \sqrt{\frac{M_{H e^{\prime}}}{M_{A^{\prime}}}} \approx \frac{v_{r o t}}{\sqrt{3}} \sqrt{\frac{M_{H e^{\prime}}}{M_{A^{\prime}}}} \mathrm{km} / \mathrm{s} \text { for } \mathrm{He}^{\prime} \text { dominated halo. }
\end{aligned}
$$

\footnotetext{
${ }^{7}$ However in the WIMP case the upper velocity limit is finite, corresponding to the galactic escape velocity. While for mirror dark matter, the upper limit is infinite due to the self interactions of the mirror particles.
} 
Mirror BBN[17] suggests that $H e^{\prime}$ dominates over $H^{\prime}$. It happens that the recoil energy thresholds of the DAMA/NaI and the other experiments are sufficiently high that these experiments are only directly sensitive to mirror elements heavier than about carbon, which means that $v_{0}\left(A^{\prime}\right) \ll v_{\text {rot }}$ for these elements - independently of whether $H e^{\prime}$ or $H^{\prime}$ dominates the halo. It turns out that our main results (such as the allowed region in figure 4) do not depend very significantly on whether we assume that $H e^{\prime}$ or $H^{\prime}$ dominates the mass of the Halo.

Finally, we see from Eq.(6) that the interaction rate, $d R / d E_{R}$, is proportional to $\epsilon^{2} n_{A^{\prime}}$, where $\epsilon$ is the photon-mirror photon kinetic mixing parameter and $n_{A^{\prime}}$ is the halo number density of the species $A^{\prime}$. The dark matter halo mass density at the Earth's location is approximately $0.3 \mathrm{GeV} / \mathrm{cm}^{3}$, so that the halo mass fraction, $\xi_{A^{\prime}}$, of the species $A^{\prime}$, is thus: $\xi_{A^{\prime}} \equiv n_{A^{\prime}} M_{A^{\prime}} /\left(0.3 \mathrm{GeV} / \mathrm{cm}^{3}\right)$. Hence, we find that the interaction rate is proportional to $\left(\epsilon \sqrt{\xi_{A^{\prime}}}\right)^{2}$. We call the quantity, $\epsilon \sqrt{\xi_{A^{\prime}}}$, the cross-section abundance coefficient, which we can determine from the amplitude of the DAMA annual modulation signal. The only other variables in the model are the rotation velocity, $v_{\text {rot }}$, which is expected to be in the range, Eq.(13), and the spectrum of heavy mirror elements, $A^{\prime}$.

\section{Mirror matter interpretation of DAMA/NaI \& DAMA/LIBRA annual modulation signal}

The DAMA experiments employ large mass scintillation detectors located in the Gran Sasso underground laboratory. The first generation, DAMA/NaI experiment ran for 7 years, presenting final results in 2003[2]. The second generation, DAMA/LIBRA experiment has been running since 2003, recently presenting its first results from 4 years of data[1].

The DAMA/NaI and DAMA/LIBRA experiments are very sensitive to mirror mattertype dark matter because of the relatively low energy threshold of 2 keVee. The unit, keVee is the so-called electron equivalent energy, which is the energy of the event if due to an electron recoil. The actual nuclear recoil energy (in $\mathrm{keV}$ ) is given by: keVee/q, where $q$ is the quenching factor.

The DAMA/NaI and DAMA/LIBRA experiments extract their signal by using the annual modulation signature[34], which arises because of the Earth's orbital motion. The point is that the interaction rate, Eq.(6), depends on $v_{E}$, which varies due to the Earth's motion around the sun:

$$
\begin{aligned}
v_{E}(t) & =v_{\odot}+v_{\oplus} \cos \gamma \cos \omega\left(t-t_{0}\right) \\
& =v_{\odot}+\Delta v_{E} \cos \omega\left(t-t_{0}\right)
\end{aligned}
$$

where $v_{\odot}=v_{\text {rot }}+12 \mathrm{~km} / \mathrm{s} \sim 230 \mathrm{~km} / \mathrm{s}$ is the sun's velocity with respect to the galactic halo and $v_{\oplus} \simeq 30 \mathrm{~km} / \mathrm{s}$ is the Earth's orbital velocity around the Sun $\left(t_{0}=152.5\right.$ days and $\omega=2 \pi / T$, with $T=1$ year). The inclination of the Earth's orbital plane relative to the galactic plane is $\gamma \simeq 60^{\circ}$, which implies that $\Delta v_{E} \simeq 15 \mathrm{~km} / \mathrm{s}$. The differential interaction rate, Eq.(6), can be expanded in a Taylor series around $v_{E}=v_{\odot}$, leading to 
an annual modulation term:

$$
R_{i}=R_{i}^{0}+R_{i}^{1} \cos \omega\left(t-t_{0}\right)
$$

where

$$
\begin{aligned}
R_{i}^{0} & =\frac{1}{\Delta E} \int_{E_{i}}^{E_{i}+\Delta E}\left(\frac{d R}{d E_{R}}\right)_{v_{E}=v_{\odot}} d E_{R} \\
R_{i}^{1} & =\frac{1}{\Delta E} \int_{E_{i}}^{E_{i}+\Delta E} \frac{\partial}{\partial v_{E}}\left(\frac{d R}{d E_{R}}\right)_{v_{E}=v_{\odot}} \Delta v_{E} d E_{R}
\end{aligned}
$$

According to the DAMA analysis[1], they indeed find an annual modulation at more than $8 \sigma$ C.L. Their data fit gives $T=(0.998 \pm 0.003)$ years and $t_{0}=144 \pm 8$ days, consistent with the expected values. [The expected value for $t_{0}$ is 152.5 days (2 June), where the Earth's velocity reaches a maximum with respect to the galactic halo]. Their signal occurs in the 2-6 keVee energy range, with amplitude

$$
R^{1}=(0.0129 \pm 0.0016) \mathrm{cpd} / \mathrm{kg} / \mathrm{keVee}[\mathrm{cpd}=\text { counts per day }]
$$

The theoretical rate for the modulated and absolute component can be evaluated in our model from Eqs.(19,6,10,11), leading to:

$$
\begin{aligned}
& \frac{d R^{0}}{d E_{R}}=\sum_{A^{\prime}} \frac{N_{T} n_{A^{\prime}} \lambda I\left(E_{R}, y_{0}\right)}{E_{R}^{2}} \\
& \frac{d R^{1}}{d E_{R}}=\sum_{A^{\prime}} \frac{N_{T} n_{A^{\prime}} \lambda \Delta y}{E_{R}^{2}}\left(\frac{\partial I}{\partial y}\right)_{y=y_{0}}
\end{aligned}
$$

where $y_{0}=\left\langle v_{E}\right\rangle / v_{0}, \Delta y=\Delta v_{E} / v_{0}$, and

$$
\left(\frac{\partial I}{\partial y}\right)_{y=y_{0}}=-\frac{I\left(E_{R}, y_{0}\right)}{y_{0}}+\frac{1}{\sqrt{\pi} v_{0} y_{0}}\left[e^{-\left(x-y_{0}\right)^{2}}+e^{-(x+y)^{2}}\right] .
$$

Recall, from Eqs. $(12,7,14)$ that $x \propto \sqrt{E_{R}}$, while $y_{0} \simeq \frac{v_{r o t}}{v_{0}}=\sqrt{\frac{M_{A^{\prime}}}{\mu M_{p}}}$, so that $y_{0} \simeq 3.4 \sqrt{\frac{M_{A^{\prime}}}{M_{O^{\prime}}}}$ $\left(5.5 \sqrt{\frac{M_{A^{\prime}}}{M_{O^{\prime}}}}\right)$ for a $H e^{\prime}\left(H^{\prime}\right)$ dominated halo.

Consider the interactions of one halo component $A^{\prime}$. What is the behaviour of the annual modulation amplitude, $d R^{1} / d E_{R}$, as a function of $E_{R}$ ? At low $E_{R}$, (where $x \ll y), d R^{1} / d E_{R}$ is negative, as $E_{R}$ increases, $d R^{1} / d E_{R}$ changes sign, and reaches a maximum when

$$
x\left(E_{R}\right) \simeq y_{0} .
$$

At high $E_{R}(x \gg y), d R^{1} / d E_{R} \rightarrow 0$.

Of course, in order to do a detailed fit of the experimental data to a given theoretical model, we must take into account the finite resolution of the DAMA detector and the 
physics of the quenching factor. In all of our numerical work both of these effects are included. The experimental energy resolution is taken into account by convolving the rate with a Gaussian, with $\sigma$ obtained from ref.[35]: $\sigma / E=\alpha / \sqrt{E(k e V)}+\beta$ with $\alpha=0.448 \pm 0.035, \beta=(9.1 \pm 5.1) \times 10^{-3}$.

The quenching factor is more complicated. Our previous work assumed $q_{N a}=0.3$ and $q_{I}=0.09$. However, recently Drobyshevski[36] has pointed out the possible importance of the 'channeling effect' in $\mathrm{NaI}$ crystals, which means that ions moving approximately in the direction of crystal axes and planes get 'channeled' whereby they give an unquenched signal, $q \simeq 1$. A detailed study and modelling of this effect has been done recently by the DAMA collaboration[15]. According to their model, the fraction of events, $f_{N a, I}$, with quenching factor $q \simeq 1$, in the low recoil energy region of interest is approximately:

$$
f_{N a} \simeq \frac{1}{1+1.14 E_{R}(k e V)}, \quad f_{I} \simeq \frac{1}{1+0.75 E_{R}(k e V)}
$$

The remaining fraction of events have quenching factors, $q_{N a} \sim 0.3, q_{I} \sim 0.09$ (but are broadened by straggling). The channeling effect is very important, as it effectively reduces the threshold of the DAMA experiments down to an actual recoil energy of 2 $\mathrm{keV}$. It turns out that the fitted cross-section is over an order of magnitude smaller compared to the case where the channeling effect is ignored. This means that the interaction rate is dominated by the channeled events.

The $2 \mathrm{keV}$ threshold is still too high for the DAMA experiment to be very sensitive to the dominant, $H e^{\prime}$ or $H^{\prime}$ component, except possibly for effects in the $E_{R} \lesssim 2.5 \mathrm{keV}$ region from the tail of the $H e^{\prime}$ induced events. Basically, these nuclei are too light to give much of a signal above the DAMA/NaI energy threshold. [Of course, the DAMA experiment is indirectly sensitive to the $H^{\prime} / H e^{\prime}$ component, which as we discussed in section 3 , has the important effect of reducing the value of $\left.v_{0}\left(A^{\prime}\right)\right]$. DAMA is only directly sensitive to mirror nuclei heavier than about carbon. In previous work we considered having several components, such as $O^{\prime}, S i^{\prime}, F e^{\prime}$ in ref.[12], however it turns out that this is not necessary to fit the new DAMA/LIBRA data. The reason is two fold: first, DAMA/LIBRA have found a lower annual modulation amplitude in the 4-6 keVee energy region than was found in the earlier DAMA/NaI experiment, second, the channeling effect also modifies the predicted spectrum. The net effect, as we will see, is that the interaction of a single halo element predicts a signal of the correct shape in the 2-6 keVee range. We therefore assume this simplest possibility, that is, that the spectrum of heavy elements (in this context 'heavy' means heavier than $M_{\mathrm{He}}$ ) is dominated by just one element, $A^{\prime}$. This would also approximate a narrow range of elements. The detailed predictions will depend on the mass of the element $A^{\prime}$, the rotational velocity $v_{\text {rot }}$ and the cross-section abundance coefficient, $\epsilon \sqrt{\xi_{A^{\prime}}}$.

Note that the cross-section abundance coefficient, $\left(\epsilon \sqrt{\xi_{A^{\prime}}}\right)^{2}$, is just an overall normalization factor, and doesn't change the shape of the annual modulation energy spectrum. This factor can be adjusted so that $R^{1}$ fits the measured amplitude averaged over the 2-6 keVee energy range. Of course, only the amplitude is a free parameter, the phase, periodicity and the approximate cosine form of the signal are all predicted, as they are 
for many other dark matter models. The fit of the annual modulation data is shown in figure 1.

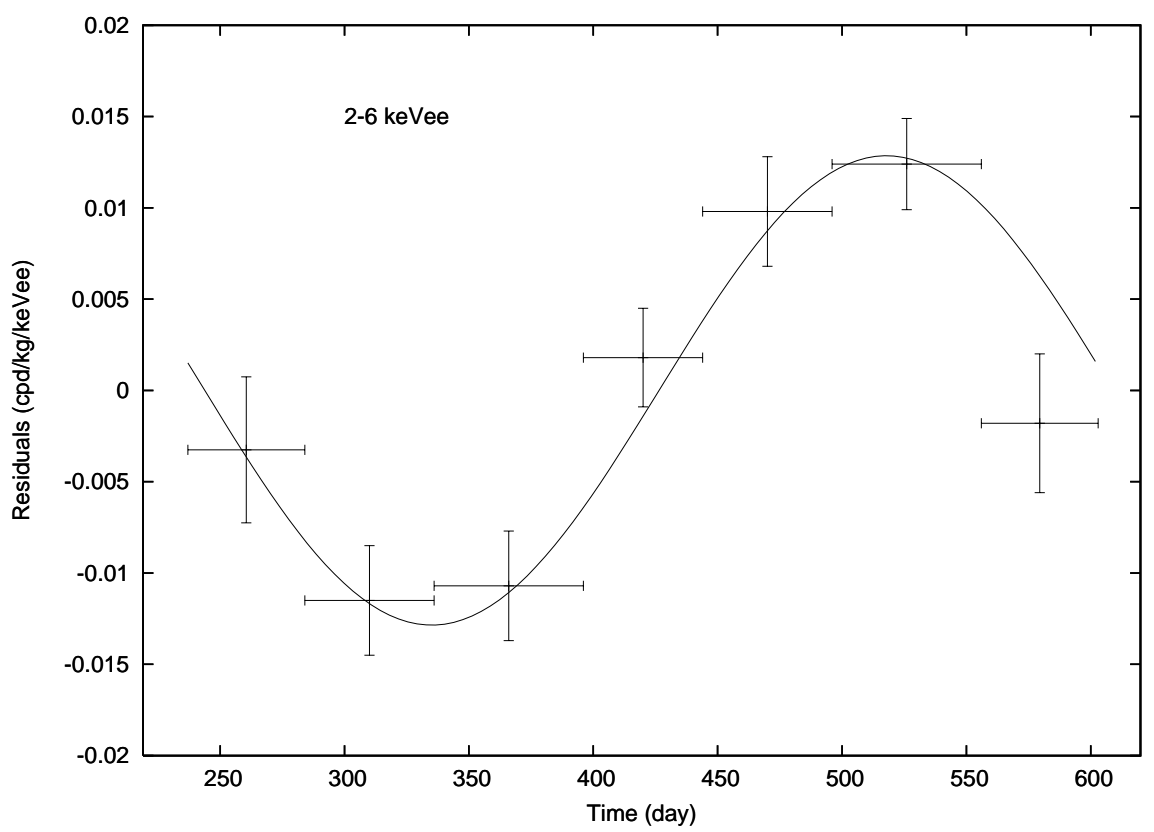

Figure 1: Measured single hit residuals for the (2-6) keVee region (obtained from figure 6 or ref.[1]) compared to the predicted cosine modulation with phase $t_{0} /$ day $=152.5$ day and $T /$ year $=1$.

An important new result from DAMA/LIBRA is that we now have a much better idea of the shape of the annual modulation energy spectrum. The DAMA collaboration separate the data in the 2-6 keVee range into 8 energy bins and fit the modulation in each energy bin to the predicted cosine distribution, obtaining modulation amplitudes, $S_{i}^{m} \pm \sigma_{i}$, for each energy bin. These amplitudes can be compared to the theoretically computed quantities, $R_{i}^{1}$, taking into account the experimental resolution and quenching factors as described earlier. We can compute a $\chi^{2}$ quantity,

$$
\chi^{2}=\sum_{i=1}^{8}\left(S_{i}^{m}-R_{i}^{1}\right)^{2} / \sigma_{i}^{2}
$$

which is a function of the three parameters: $v_{r o t}, \epsilon \sqrt{\xi_{A^{\prime}}}$ and the identity of the dominant element $A^{\prime}$. Thus, with eight independent data bins we have five degrees of freedom. We find numerically that for each value of $v_{\text {rot }}$ [in the range, Eq.(13)], the model provides a good fit to the data, with:

$$
\begin{aligned}
& \chi_{\min }^{2} \approx 5.5, \text { for } \mathrm{H}^{\prime} \text { dominated halo } \\
& \chi_{\min }^{2} \approx 3.5, \text { for } \mathrm{He}^{\prime} \text { dominated halo }
\end{aligned}
$$


for five degrees of freedom. The data does constrain the other two parameters. Numerically we obtain the $3 \sigma$ range for $\epsilon \sqrt{\xi_{A^{\prime}}}$ :

$$
|\epsilon| \sqrt{\frac{\xi_{A^{\prime}}}{0.1}} \simeq(1.0 \pm 0.3) \times 10^{-9}\left(\frac{v_{r o t}}{210 \mathrm{~km} / \mathrm{s}}\right)^{\frac{7}{4}}
$$

for both $H^{\prime}$ and $H e^{\prime}$ dominated halo.

The allowed range for $A^{\prime}$ also depends on $v_{\text {rot }}$. We find that we can fit the DAMA data for $A^{\prime}$ ranging in mass from oxygen to sulfur, i.e. $15 \mathrm{GeV} \lesssim M_{A^{\prime}} \lesssim 30 \mathrm{GeV}$. In figure 2 we have given the best fits for four illustrative examples, $A^{\prime}=0^{\prime}$, $v_{\text {rot }}=280$ $\mathrm{km} / \mathrm{s}, A^{\prime}=N e^{\prime}, v_{\text {rot }}=230 \mathrm{~km} / \mathrm{s}, A^{\prime}=M g^{\prime}, v_{\text {rot }}=195 \mathrm{~km} / \mathrm{s}$ and $A^{\prime}=S i^{\prime}, v_{\text {rot }}=170$ $\mathrm{km} / \mathrm{s}$. Figure $2 \mathrm{a}$ assumes a $H^{\prime}$ mass dominated halo, while figure $2 \mathrm{~b}$ assumes a $H e^{\prime}$ mass dominated halo.

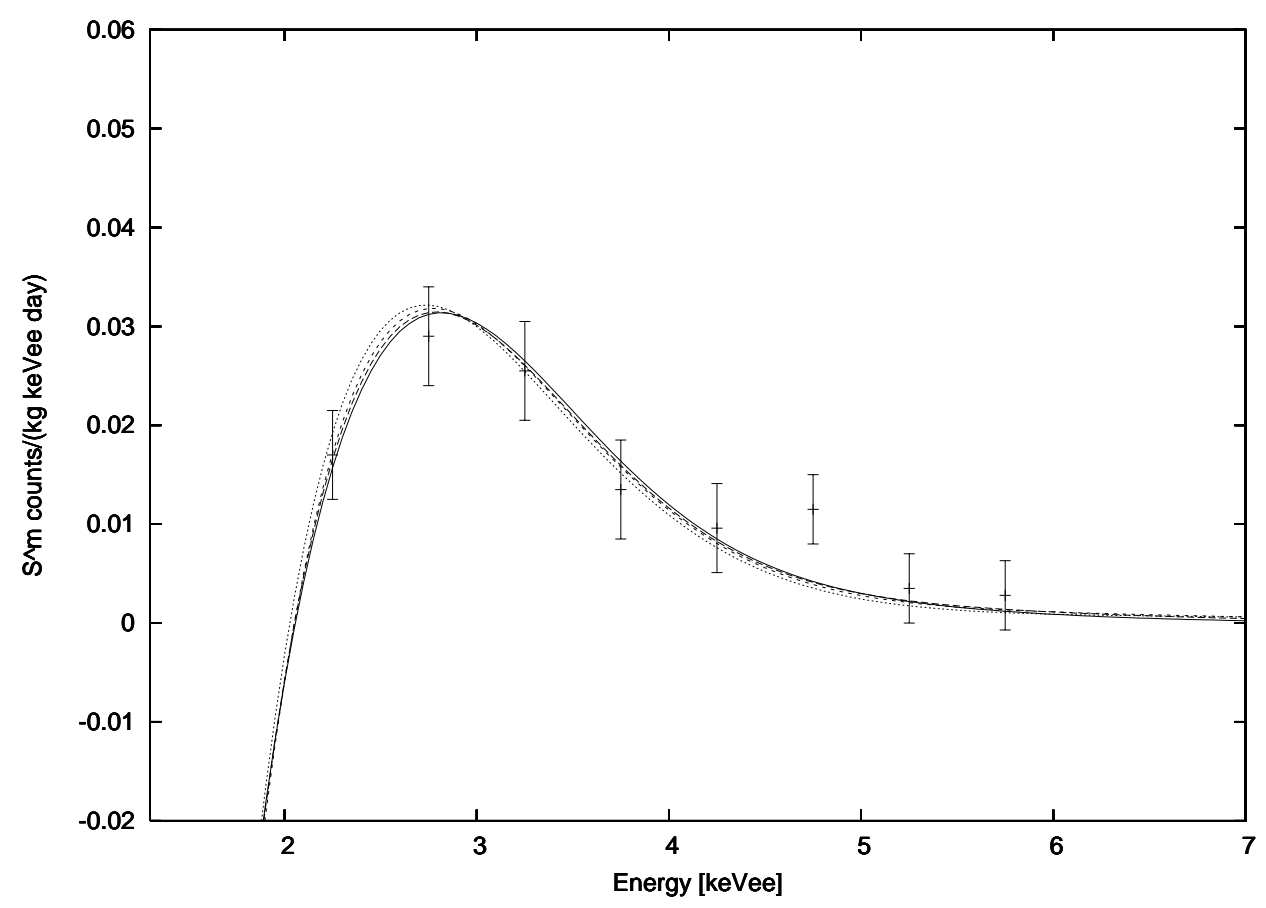

Figure 2a: Energy distribution of the cosine modulation amplitude for four illustrative cases: $A^{\prime}=S i^{\prime}, v_{\text {rot }}=170 \mathrm{~km} / \mathrm{s}$ (solid line) $A^{\prime}=M g^{\prime}, v_{\text {rot }}=195 \mathrm{~km} / \mathrm{s}$ (long-dashed line), $A^{\prime}=N e^{\prime}, v_{r o t}=230 \mathrm{~km} / \mathrm{s}$ (short-dashed line), $A^{\prime}=O^{\prime}, v_{r o t}=280 \mathrm{~km} / \mathrm{s}$ (dotted line). In each case $\epsilon \sqrt{\xi_{A^{\prime}}}$ is fixed so that the mean amplitude is $0.0129 \mathrm{cpd} / \mathrm{kg} / \mathrm{keVee}$. Also shown are the DAMA/NaI \& DAMA/LIBRA combined data from figure 9 of ref.[1]. This figure assumes that the mass of the halo is dominated by $H^{\prime}$. 


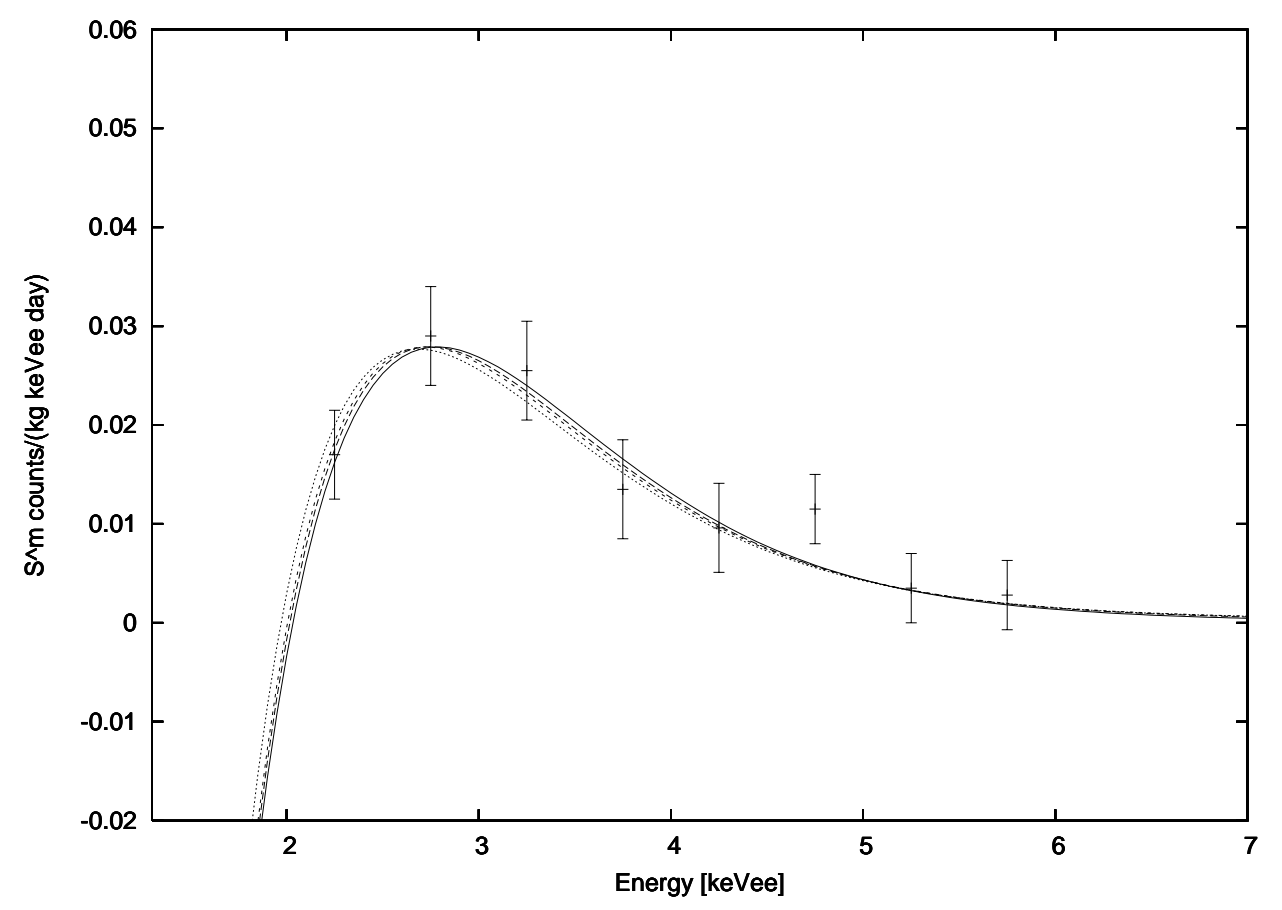

Figure 2b: Same as figure 2a, except the mass of the halo is assumed to be dominated by $H e^{\prime}$.

The shape of the measured annual modulation energy spectrum agrees very well with the mirror dark matter theory. It is essentially a two parameter fit, since as figure 2 demonstrates, there is an approximate redundancy. Once the height and position of the peak is fitted, there is little freedom left to change the shape of the distribution. Thus, the fact that the measured shape agrees with that predicted by the mirror matter theory is a significant test of the theory. We illustrate this in figure 3 by fixing $v_{\text {rot }}$ and varying $M_{A^{\prime}}$ around the best fit value, for a particular example. 


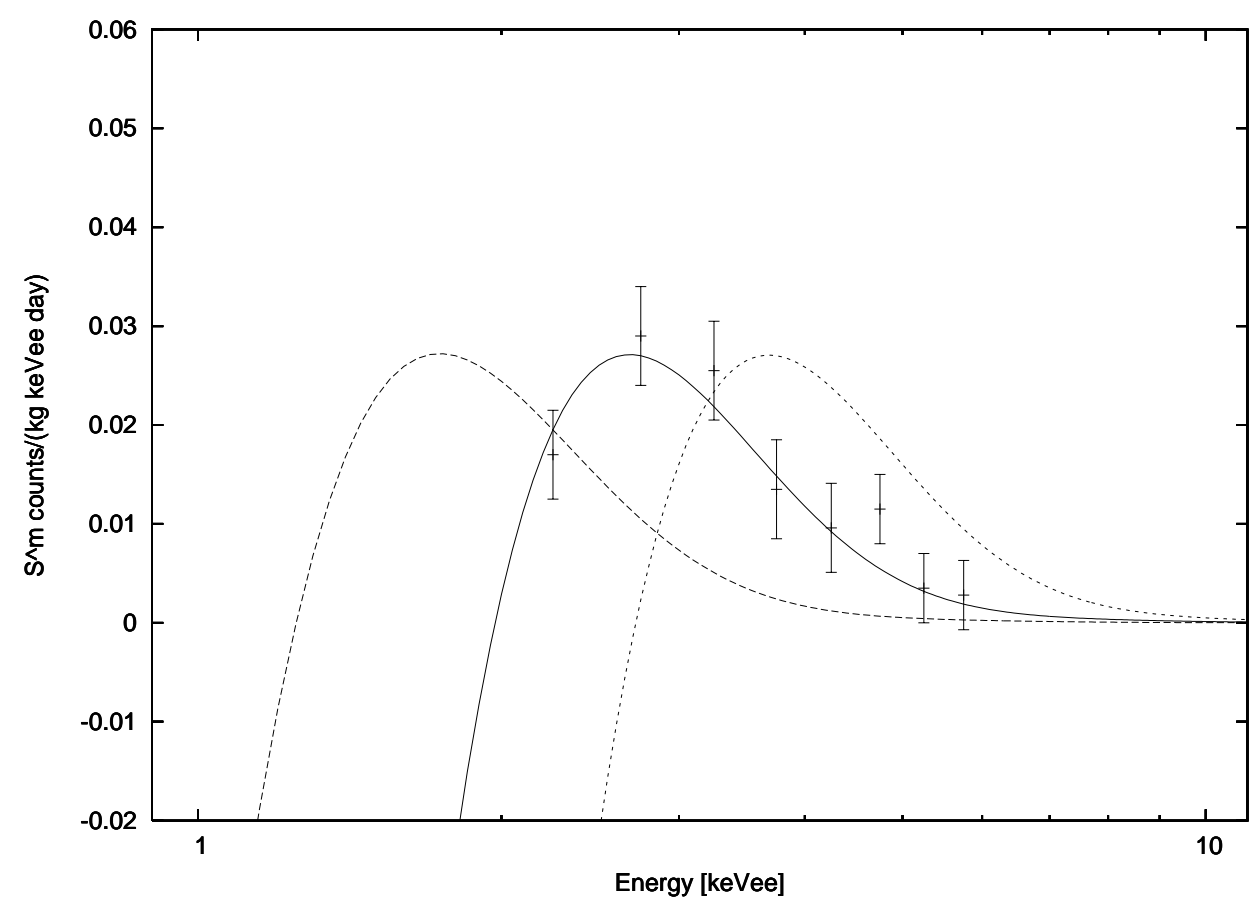

Figure 3: Annual modulation amplitude for the case of $A^{\prime}=O^{\prime}$, (solid line), $A^{\prime}=C^{\prime}$ (dashed line), $A^{\prime}=N e^{\prime}$ (short-dashed line), ie. with $A^{\prime}=16 \pm 4$. In each case we have used the same $v_{\text {rot }}=280 \mathrm{~km} / \mathrm{s}$, assumed a $H e^{\prime}$ mass dominated halo, and normalized the height of the peak to be the same. This figure illustrates that the position of the peak determines $A^{\prime}$, with the shape of the energy spectrum being approximately unchanged.

As figure 3 illustrates, the position of the peak provides a measurement of the mass of the element $A^{\prime}$. Allowing for the possible uncertainty in $v_{\text {rot }}$, pins down the mass range for the element $A^{\prime}$ to the range: $15 \mathrm{GeV} \lesssim M_{A^{\prime}} \lesssim 30 \mathrm{GeV}$. It is interesting that this mass range is theoretically consistent $\left(M_{\mathrm{He}}<M_{A^{\prime}}<M_{\mathrm{Fe}}\right)$ and also close to the naive expectation, of oxygen mass. Oxygen is the most abundant heavy ordinary element in the Universe, and it would not be surprising if the mirror sector were similar.

If we consider $A^{\prime}$ as a parameter, then we can evaluate the $3 \sigma(5 \sigma)$ DAMA allowed region by finding the contours in $\left(v_{\text {rot }}, A^{\prime}\right)$ space where $\chi^{2}=\chi_{\min }^{2}+9\left(\chi^{2}=\chi_{\min }^{2}+25\right)$, where at each point, we vary $\epsilon \sqrt{\xi_{A^{\prime}}}$ such that the normalization is correct. We plot this DAMA allowed region in figure $\mathbf{4}$, for the case of a $H e^{\prime}$ mass dominated halo. The case of a $H^{\prime}$ dominated halo is very similiar. 


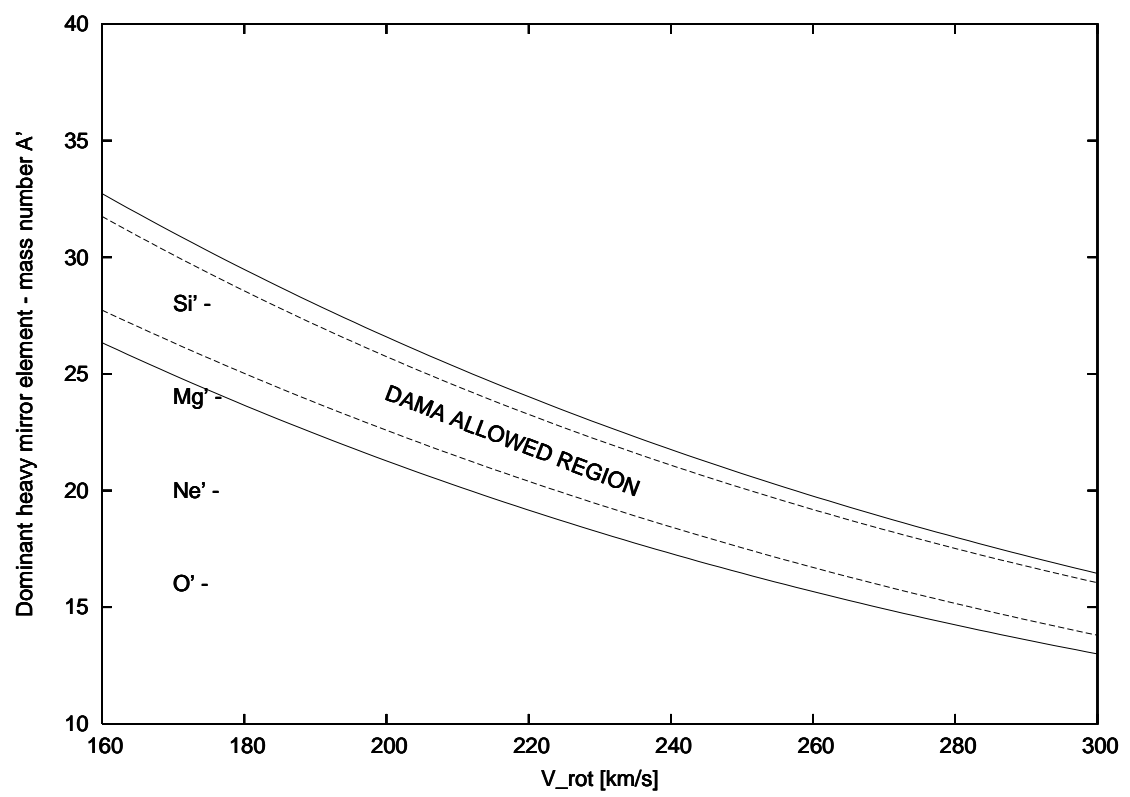

Figure 4: The $3 \sigma$ (dashed line) and $5 \sigma$ (solid line) allowed region of $v_{r o t}, A^{\prime}$ parameter space consistent with the measured energy distribution of the modulation amplitude. In each case we have fixed $\epsilon \sqrt{\xi_{A^{\prime}}}$ so that the mean modulation amplitude is 0.0129 $\mathrm{cpd} / \mathrm{kg} / \mathrm{keVee}$.

The main features of our numerical results can be approximately understood: The data shows evidence for a peak in the annual modulation energy spectrum. A peak is expected analytically. As we discussed earlier [Eq.(23)], the position of the peak corresponds to the value of $E_{R}$ (which we denote as $E_{R}^{\text {peak }}$ ) where $x \simeq y_{0}$, or equivalently, to the value of $E_{R}$ where $v_{\text {min }}\left(E_{R}^{\text {peak }}\right) \simeq v_{\text {rot }}$. Evaluating this expression, using Eq.(7), we find:

$$
M_{A^{\prime}} \simeq \frac{M_{A}}{v_{\text {rot }} \sqrt{\frac{2 M_{A}}{E_{R}^{\text {pack }}}}-1}
$$

This shows again that the value of $E_{R}$ at the maximum of $d R^{1} / d E_{R}$, provides a determination of the mass of the dominant heavy mirror element $A^{\prime}$. The experimental data suggests that the maximum occurs roughly at $E_{R} \simeq 2.75 \mathrm{keVee}$. Evaluating Eq.(28) for $A=I$ and for $A=N a$ gives:

$$
\begin{aligned}
& \frac{M_{A^{\prime}}}{M_{p}} \simeq \frac{13.7}{\left(\frac{v_{r o t}}{300 k m / s}\right)-0.107}, \text { for } \mathrm{A}=\mathrm{I} \\
& \frac{M_{A^{\prime}}}{M_{p}} \simeq \frac{5.8}{\left(\frac{v_{r o t}}{300 k m / s}\right)-0.25}, \text { for } \mathrm{A}=\mathrm{Na}
\end{aligned}
$$

Thus, we see that our allowed region corresponds to the case where $A^{\prime}$ is interacting predominately with $I$. We might have expected a second distinct region of allowed 
parameter space - a second branch at lower $v_{\text {rot }}$ - where the rate is dominated by interactions with $N a$. Numerically it turns out that the $N a$ peak is washed out by the interactions of $I$, whose peak then occurs around 1.1 keVee (if the peak in $N a$ is at 2.75 keVee), and is smeared out into the $E>2$ keVee range by the detector resolution. The height of the $I$ peak is also enhanced c.f. with $N a$ peak, because a) the basic crosssection on $I$ is larger than $N a$ because of the larger $I$ nuclear electric charge, b) the $I$ peak occurs at lower $E_{R}$ and $\frac{d \sigma}{d E_{R}} \propto \frac{1}{E_{R}^{2}}$, c) the fraction of channeled events strongly increases towards unity as $E_{R} \rightarrow 0$ in the adopted quenching model of ref.[15].

Finally, in figure 5, we give the absolute (unmodulated) spectrum, predicted by the model, and compare it to the DAMA spectrum. Of course, the measured absolute spectrum has a significant background component which is removed when the time varying component is extracted. Figure 5 indicates that in this model the required background spectrum is roughly consistent - it is smooth and positive. There is also a hint of the large rise in absolute rate predicted by the model at low energies. Of course, this is below the experimental threshold, and this hint should not be taken too seriously.

Note that because of the sharply rising absolute spectrum at low $E_{R}$ our predicted shape of the absolute spectrum is somewhat sensitive to uncertainties in detector resolution and quenching factor. The shape of the modulation spectrum, on the other hand, is much less sensitive to these uncertainties.

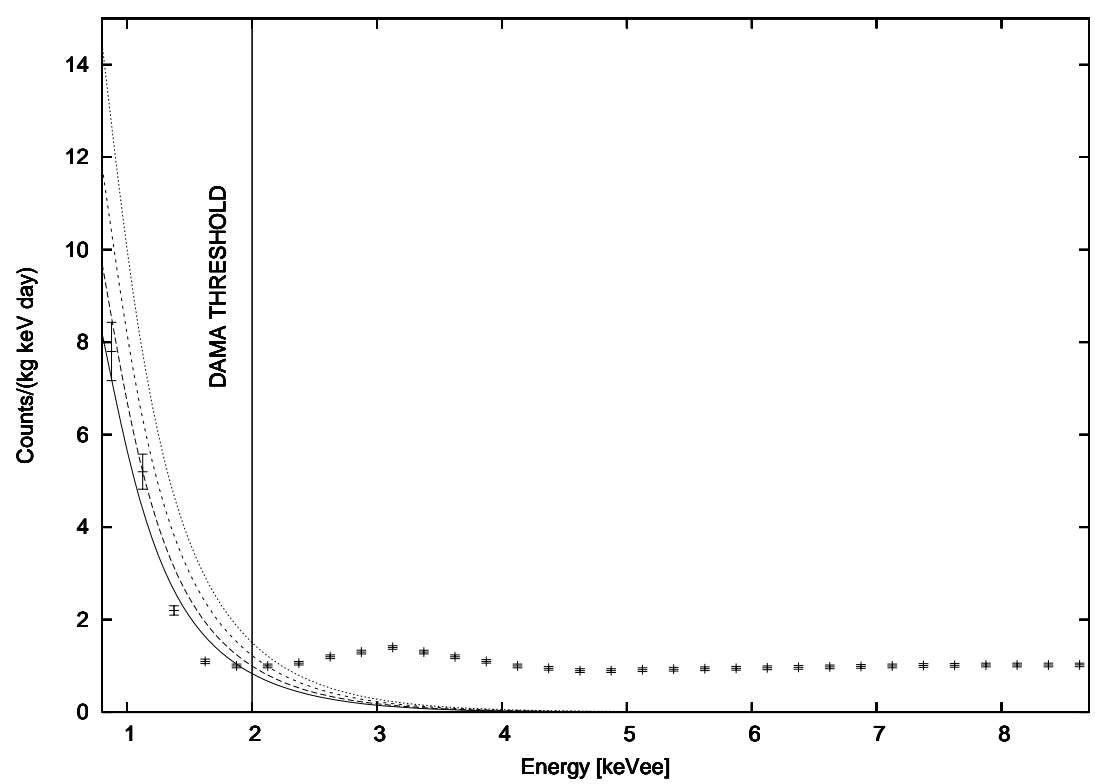

Figure 5a: Predicted absolute (unmodulated) rate, $\frac{d R^{0}}{d E_{R}}$, for the DAMA/NaI experiment. We take the same four examples of figure $2 \mathrm{a}: A^{\prime}=S i^{\prime}, v_{r o t}=170 \mathrm{~km} / \mathrm{s}$ (solid line) $A^{\prime}=M g^{\prime}, v_{r o t}=195 \mathrm{~km} / \mathrm{s}$ (long-dashed line), $A^{\prime}=N e^{\prime}, v_{r o t}=230 \mathrm{~km} / \mathrm{s}$ (short-dashed line), $A^{\prime}=O^{\prime}, v_{\text {rot }}=280 \mathrm{~km} / \mathrm{s}$ (dotted line). As with figure $2 \mathrm{a}$, these results assume a $H^{\prime}$ dominated halo by mass. Also shown is the experimental (single hit) data. 


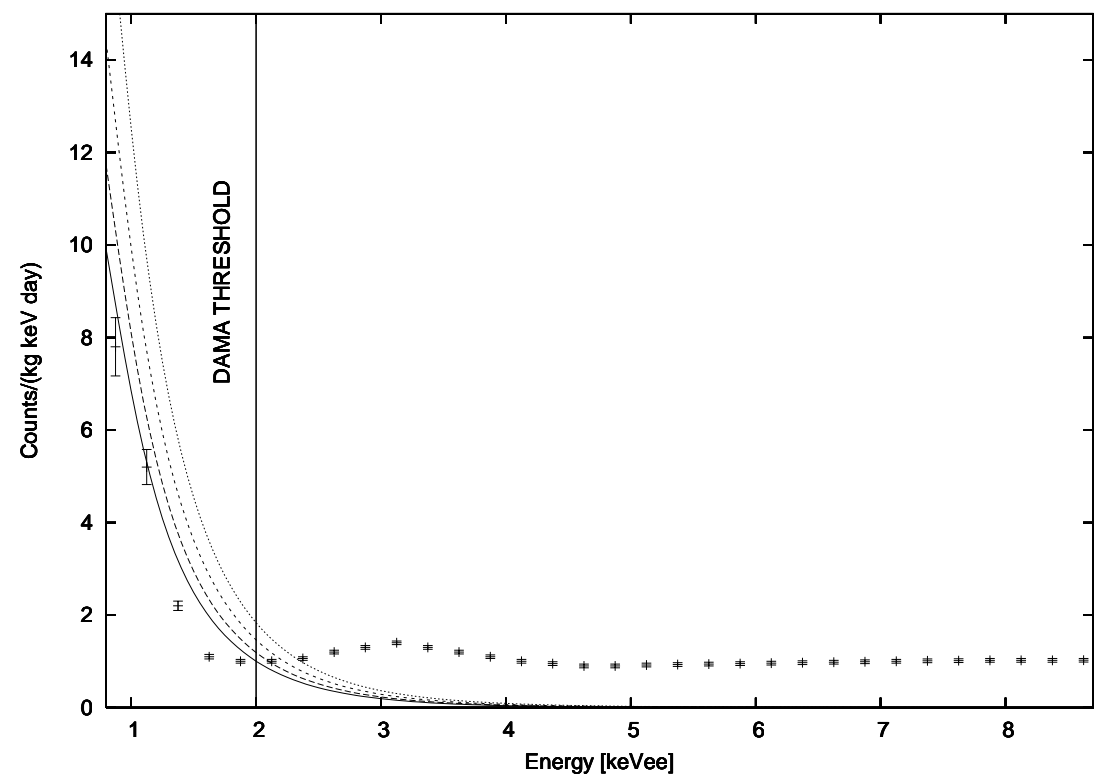

Figure 5b: Same as figure 5a, except the mass of the halo is assumed to be dominated by $H e^{\prime}$.

The value of $\epsilon \sqrt{\xi_{A^{\prime}}}$ obtained from the fit to the DAMA/NaI and DAMA/LIBRA experiments suggests a value for $\epsilon$ in the range $10^{-9}-10^{-8}$ for $10^{-3}<\xi_{A^{\prime}}<10^{-1}$. Values of $\epsilon$ in this range have many interesting applications, see e.g. ref.[37, 38]. A mirror sector, interacting with the ordinary sector, with kinetic mixing in this range is also consistent with Laboratory[39] and big bang nucleosynthesis constraints[40].

\section{Constraints from the null experiments: CDMS/Ge, CDMS/Si, XENON10}

The annual modulation signal has only been seen in the DAMA/NaI and DAMA/LIBRA experiments. Other direct detection experiments have not found any dark matter signal. The most sensitive of these experiments are the CDMS and XENON10 experiments. These experiments use sensitive background elimination techniques and place limits on the absolute dark matter event rate, above their recoil energy detection thresholds.

Importantly, these experiments are all higher threshold experiments, with claimed nuclear recoil energy thresholds of $10 \mathrm{keV}$ for CDMS/Ge, $7 \mathrm{keV}$ for CDMS/Si and 4.5 $\mathrm{keV}$ for XENON10. The fact that mirror dark matter is light $\left(M_{A^{\prime}} \sim M_{O}\right)$ has a narrow velocity dispersion, $v_{O}\left(A^{\prime}\right)^{2} \ll v_{r o t}^{2}$, and has cross-section proportional to $1 / E_{R}^{2}$ all help to enhance the signal in DAMA/NaI relative to these higher threshold experiments.

We have evaluated the contraints on our model from the CDMS/Ge[3], CDMS/Si[4] and XENON10[5] experiments. The published net effective exposures for these experiments are $121 \mathrm{~kg}-d, 12 \mathrm{~kg}-d$ and $\sim 100 \mathrm{~kg}-d$ for CDMS/Ge, CDMS/Si and XENON 
respectively. As, indicated by figure 5, we expect a sharply falling absolute event rate. Therefore the most sensitive energy region in these higher threshold experiments will be near their energy threshold. In the threshold region, CDMS/Ge[3], CDMS/Si[4] and XENON10[5] found 0, 0 and 1 event respectively. The Poisson 95\% exclusion region is then $N=3$ for the CDMS experiments and $N=4.74$ for the XENON experiment. We have computed these exclusion limits for the parameter space of interest for the DAMA experiment, which we show in figure 6. As the figure clearly demonstrates, our favoured region of parameter space is consistent with the null results of these experiments.

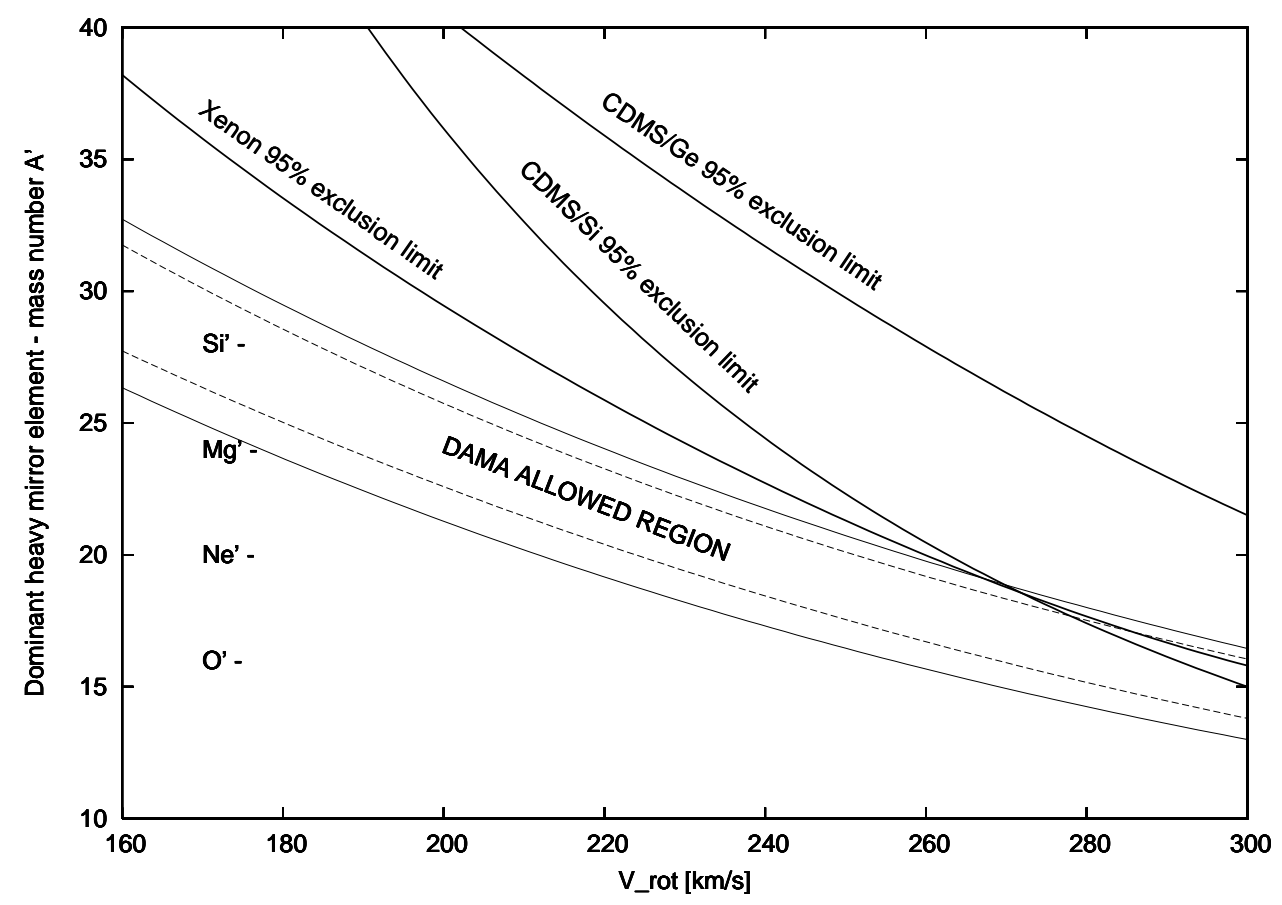

Figure 6: DAMA allowed region, as shown in figure 4, except with the addition of the 95\% C.L. exclusion limits from the XENON10, CDMS/Ge and CDMS/Si experiments (the regions above the exclusion contours are the disfavoured region).

Finally, note that there are a few experiments with lower thresholds than DAMA/NaI. In particular there is the CRESSTI experiment with threshold of $0.6 \mathrm{keV}[41]$. The compatibility of the CRESST experiment with the DAMA/NaI annual modulation signal was analysed in the mirror matter context in ref.[9], but without taking into account the channeling effect in the DAMA analysis. It was found that the predicted rate for the CRESST experiment was about a factor of two higher than the measured rate. Including the channeling effect in the DAMA analysis lowers the predicted cross section by more than an order of magnitude, which means that the predicted event rate for the CRESST experiment is around $10 \%$ of their measured event rate. Thus the mirror dark matter interpretation of the DAMA/NaI signal is completely consistent with 
the results of the low threshold CRESST experiment. Another low threshold experiment of note is the TEXONO experiment[42] with a claimed threshold of $0.2 \mathrm{keV}$. The low exposure and large background currently limit the usefulness of that experiment. However, the sharply rising event rate at low recoil energies, predicted by the mirror dark matter explanation of the DAMA/NaI experiment, might potentially be probed by future upgrades of experiments such as TEXONO and CRESST. The possibility of searching for annual modulation in such low threshold experiments would, of course, also be worthwhile.

Finally note that there are earlier CDMS/Ge experiments with thresholds of 5 $\mathrm{keV}[43]$ and $7 \mathrm{keV}[4]$. However the lower exposure and high backgrounds of these early runs make these experiments less sensitive than the XENON10 experiment for the mirror dark matter interpretation of the DAMA experiment, and therefore, within this theoretical framework, they do not disfavour any of the DAMA allowed parameter space.

\section{$6 \quad$ Hidden sector dark matter models}

Mirror matter arises if one assumes an extra sector of particles and forces exactly isomorphic to the known particles. With the assumption that $T^{\prime} \ll T$ in the early Universe, this simple model is compatible with the inferred properties of dark matter, such as its large scale structure, and, as we have shown, with all of the direct detection experiments as well.

Of course, one can imagine generic hidden sector models which can approximately mimic the effects of the mirror matter model for dark matter experiments. The simplest such model would have a) two stable particles with masses $M_{1}$ and $M_{2}$, such that $M_{1} \ll M_{2}$ and b) interact with each other via an unbroken $U(1)^{\prime}$ (and possibly other gauge interactions), and c) interact with ordinary matter via $U(1)_{Y} \otimes U(1)^{\prime}$ kinetic mixing. With these assumptions, one can have $v_{0}\left(M_{2}\right)^{2} \ll v_{\text {rot }}^{2}$ if the mass density of the halo is dominated by the lighter particles of mass $M_{1}$. Provided that $15 \mathrm{GeV} \lesssim M_{2} \lesssim 30$ $\mathrm{GeV}$ such a model can give similar predictions to the mirror matter theory, and thus is experimentally viable - at the moment.

However it is interesting that all these features automatically occur in the mirror matter theory, which is theoretically tightly constrained. For example, in the mirror matter theory, $M_{2}$ is predicted to be in the range $M_{H e}<M_{A^{\prime}} \leq M_{F e}$. Thus, the mirror dark matter theory is theoretically favoured over some generic alternative hidden sector model. This theoretical prejudice will be put to the test by future experiments, which we await with interest.

\section{Conclusion}

Mirror dark matter arises in simple and renormalizable extensions of the standard model whereby an exactly isomorphic sector of particles and forces is hypothesized which interact with the known particles via renormalizable $U(1)$ kinetic mixing. Such extensions 
of the standard model are theoretically well motivated, allowing space-time parity to exist as an exact unbroken symmetry. They also supply a spectrum of necessarily stable dark matter candidates of known masses.

We have demonstrated that this dark matter theory can simply and fully explain the annual modulation signal seen in the DAMA/NaI and DAMA/LIBRA experiments. Our results indicate that the simplest and most plausible scenario: a predominant $H^{\prime} / H e^{\prime}$ halo with a small subdominant $\sim O^{\prime}$ component is sufficient to explain the data. The required photon-mirror photon mixing parameter is $\sim 10^{-9}$, which is consistent with all other experiments, and observations. In particular, we have shown that this simple scenario is also completely compatible with the latest null results of the other experiments, such as CDMS and XENON10.

Generic hidden sector models which approximately mimic the effects of mirror matter type dark matter for direct detection experiments are also possible, but theoretically less appealing. Future experiments will, of course, be the final arbitrator.

\section{Acknowledgements}

This work was supported by the Australian Research Council.

\section{References}

[1] R. Bernabei et al. (DAMA Collaboration), arXiv:0804.2741 (2008).

[2] R. Bernabei et al. (DAMA Collaboration), Phys. Lett. B480, 23 (2000); Riv. Nuovo Cimento. 26, 1 (2003) [astro-ph/0307403]; Int. J. Mod. Phys. D13, 2127 (2004) and references there-in.

[3] Z. Ahmed et al. (CDMS Collaboration), arXiv: 0802.3530 (2008).

[4] D. S. Akerib et al. (CDMS Collaboration), Phys. Rev. Lett. 96, 011302 (2006) [arXiv: astro-ph/0509259].

[5] J. Angle et al. (XENON Collaboration), Phys. Rev. Lett. 100, 021303 (2008) [arXiv:0706.0039].

[6] P. Gondolo and G. Gelmini, Phys. Rev. D71, 123520 (2005) [arXiv: hep$\mathrm{ph} / 0504010]$.

[7] R. Bernabei et al. (DAMA Collaboration), Int. J. Mod. Phys. A21, 1445 (2006) [arXiv:astro-ph/0511262].

[8] R. Bernabei et al. (DAMA Collaboration), arXiv:0802.4336 (2008).

[9] R. Foot, Phys. Rev. D69, 036001 (2004) [hep-ph/0308254].

[10] R. Foot, astro-ph/0403043. 
[11] R. Foot, Mod. Phys. Lett. A19, 1841 (2004) [astro-ph/0405362].

[12] R. Foot, Phys. Rev. D74, 023514 (2006) [arXiv: astro-ph/0510705].

[13] R. Foot, Int. J. Mod. Phys. D13, 2161 (2004) [astro-ph/0407623].

[14] R. Foot, H. Lew and R. R. Volkas, Phys. Lett. B272, 67 (1991). The idea of a mirror sector was discussed, prior to the advent of the standard model, in: T. D. Lee and C. N. Yang, Phys. Rev. 104, 256 (1956); I. Kobzarev, L. Okun and I. Pomeranchuk, Sov. J. Nucl. Phys. 3, 837 (1966). The idea that the mirror particles might be the dark matter was first discussed in: S. I. Blinnikov and M. Yu. Khlopov, Sov. J. Nucl. Phys. 36, 472 (1982); Sov. Astron. 27, 371 (1983).

[15] R. Bernabei et al. (DAMA Collaboration), arXiv: 0710.0288 (2007).

[16] E. W. Kolb, D. Seckel and M. S. Turner, Nature 314, 415 (1985); H. Hodges, Phys. Rev. D47, 456 (1993); Z. G. Berezhiani, A. D. Dolgov and R. N. Mohapatra, Phys. Lett. B375, 26 (1996) [hep-ph/9511221].

[17] Z. Berezhiani, D. Comelli and F. L. Villante, Phys. Lett. B503, 362 (2001) [hep$\mathrm{ph} / 0008105]$.

[18] A. Yu. Ignatiev and R. R. Volkas, Phys. Rev. D68, 023518 (2003) [hep$\mathrm{ph} / 0304260]$.

[19] Z. Berezhiani, P. Ciarcelluti, D. Comelli and F. L. Villante, Int. J. Mod. Phys. D14, 107 (2005) [astro-ph/0312605]; P. Ciarcelluti, Int. J. Mod. Phys. D14, 187 (2005) [astro-ph/0409630]; Int. J. Mod. Phys. D14, 223 (2005) [astro-ph/0409633].

[20] L. Bento and Z. Berezhiani, Phys. Rev. Lett. 87, 231304 (2001) [hep-ph/0107281]; hep-ph/0111116.

[21] R. Foot and R. R. Volkas, Phys. Rev. D68, 021304 (2003) [hep-ph/0304261]; Phys. Rev. D69, 123510 (2004) [hep-ph/0402267].

[22] See e.g. Z. Silagadze, Phys. Atom. Nucl. 60, 272 (1997) [hep-ph/9503481].

[23] S. Calchi Novati, II Nuovo Cimento 122B, 557 (2007) [arXiv:0711.4474].

[24] R. Foot and R. R. Volkas, Phys. Rev. D70, 123508 (2004) [astro-ph/0407522].

[25] Z. Berezhiani, P. Ciarcelluti, S. Cassisi and A. Pietrinferni, astropart. Phys. 24, 495 (2006) [astro-ph/0507153].

[26] R. Foot and X-G. He, Phys. Lett. B267, 509 (1991).

[27] R. Foot, H. Lew and R. R. Volkas, Mod. Phys. Lett. A7, 2567 (1992); R. Foot, Mod. Phys. Lett. A9, 169 (1994) [hep-ph/9402241]; R. Foot and R. R. Volkas, Phys. Rev. D52, 6595 (1995) [hep-ph/9505359]. 
[28] B. Holdom, Phys. Lett. B166, 196 (1986).

[29] R. Foot, A. Yu. Ignatiev and R. R. Volkas, Phys. Lett. B503, 355 (2001) [astro$\mathrm{ph} / 0011156]$.

[30] R. Foot, Int. J. Mod. Phys. A19 3807 (2004) [astro-ph/0309330].

[31] R. H, Helm, Phys. Rev. 104, 1466 (1956).

[32] J. D. Lewin and P. F. Smith, Astropart. Phys. 6, 87 (1996).

[33] C. S. Kochanek, Astrophys. J. 457, 228 (1996).

[34] A. K. Drukier, K. Freese and D. N. Spergel, Phys. Rev. D33, 3495 (1986); K. Freese, J. A. Frieman and A. Gould, Phys. Rev. D37, 3388 (1988).

[35] R. Bernabei et al. (DAMA Collaboration), arXiv: 0804.2738 (2008).

[36] E. M. Drobyshevski, arXiv: 0706.3095.

[37] R. Foot and S. Mitra, Astropart. Phys. 19, 739 (2003) [astro-ph/0211067]; Phys. Lett. B558, 9 (2003) [hep-ph/0303005]; Phys. Lett. A315, 178 (2003) [condmat/0306561].

[38] R. Foot and Z. K. Silagadze, Int. J. Mod. Phys. D14, 143 (2005) [astro$\mathrm{ph} / 0404515]$.

[39] S. L. Glashow, Phys. Lett. B167, 35 (1986); R. Foot and S. N. Gninenko, Phys. Lett. B480, 171 (2000) [hep-ph/0003278]. Although the current best limit on $\epsilon$ from laboratory experiments is[30] $\epsilon<5 \times 10^{-7}$, forthcoming orthopositronium experiments [A. Badertscher et al., Int. J. Mod. Phys. A19, 3833 (2004)] may probe the interesting region $\epsilon \sim 10^{-9}$.

[40] E. D. Carlson and S. L. Glashow, Phys. Lett. B193, 168 (1987).

[41] G. Angloher it et al. (CRESST Collaboration), Astroparticle Phys. 18, 43 (2002).

[42] S. T. Lin et al. (TEXONO Collaboration), arXiv:0712.1645

[43] D. S. Akerib et al. (CDMS Collaboration) Phys. Rev. D68: 082002 (20030 [arXiv: hep-ex/0306001]. 